\title{
MINIMIZING THE LAPLACIAN OF A FUNCTION SQUARED WITH PRESCRIBED VALUES ON INTERIOR BOUNDARIES- THEORY OF POLYSPLINES
}

\author{
OGNYAN IV. KOUNCHEV
}

\begin{abstract}
In this paper we consider the minimization of the integral of the Laplacian of a real-valued function squared (and more general functionals) with prescribed values on some interior boundaries $\Gamma$, with the integral taken over the domain $\mathrm{D}$. We prove that the solution is a biharmonic function in $D$ except on the interior boundaries $\Gamma$, and satisfies some matching conditions on $\Gamma$. There is a close analogy with the one-dimensional cubic splines, which is the reason for calling the solution a polyspline of order 2 , or biharmonic polyspline. Similarly, when the quadratic functional is the integral of $\left(\Delta^{q} f\right)^{2}, q$ a positive integer, then the solution is a polyharmonic function of order $2 q, \Delta^{2 q} f(x)=0$, for $x \in D \backslash \Gamma$, satisfying matching conditions on $\Gamma$, and is called a polyspline of order $2 q$. Uniqueness and existence for polysplines of order $2 q$, provided that the interior boundaries $\Gamma$ are sufficiently smooth surfaces and $\partial D \subseteq \Gamma$, is proved. Three examples of data sets $\Gamma$ possessing symmetry are considered, in which the computation of polysplines is reduced to computation of onedimensional $L$-splines.
\end{abstract}

\section{INTRODUCTION}

In the cases when the values of the function or its derivatives are prescribed on the boundary of the domain, the problem of minimizing a quadratic functional is reduced to the integration of a differential equation under appropriate boundary conditions. That is a fundamental fact for the classical variational calculus [Mikh].

In the present paper we consider the minimization of a quadratic functional when the values of the function are prescribed on interior boundaries of codimension 1 . We will work in a greater generality than indicated in the title of the paper, but all important features of the theory presented here occur in the simplest case, when the quadratic functional is given by the square of the Laplacian of a function. This is the simplest nontrivial example which illustrates all features.

We will explain the ideas underlying the present paper by analogy with onedimensional spline theory.

Received by the editors January 19, 1993 and, in revised form, September 17, 1996.

1991 Mathematics Subject Classification. Primary 35J40; Secondary 41A15, 65D07.

Key words and phrases. Polyharmonic functions, a priori estimates, multivariate splines, variational problem.

Partially sponsored by the Alexander von Humboldt Foundation and by the NFSR of the Bulgarian Ministery of Education and Science under grant number MM21/91.

(C) 1998 American Mathematical Society 
The interpolation splines of one variable may be considered as solutions to the following extremal problem:

$$
\int_{a}^{b}\left[d^{q} f(t) / d t^{q}\right]^{2} d t \rightarrow \inf
$$

over the set of functions which satisfy the interpolation conditions:

$$
\begin{gathered}
f\left(t_{j}\right)=c_{j}, \quad j=1,2, \ldots, N \\
d^{k} f(t) / d t_{\mid t=a}^{k}=d^{k} f(t) / d t_{\mid t=b}^{k}=0,
\end{gathered}
$$

for $k=1, \ldots, q-1$, where $a=t_{1}<\ldots<t_{N}=b$.

Notice that we do not use the very standard definition of natural splines in [Laur, p. 156] which requires $N \geq q$, but rather that in [ANW, p. 109].

The solution to problem $(1.1)-\left(1.2^{\prime}\right)$ is a function $s(t)$ in the interval $[a, b]$ which satisfies the following conditions:

(i) $s(t)$ is a polynomial of degree $\leq 2 q-1$ in every open interval $\left(t_{i}, t_{i+1}\right), i=$ $1,2, \ldots, N-1$, i.e. $d^{2 q} s(t) / d t^{2 q}=0$ for all $t \in\left(t_{i}, t_{i+1}\right)$.

(ii) The following boundary conditions hold: $d^{k} s(a) / d t^{k}=d^{k} s(b) / d t^{k}=0$, for every $k=1, \ldots, q-1$.

(iii) $d^{2 q-2} s(t) / d t^{2 q-2}$ is continuous everywhere in $[a, b]$, i.e.

$$
d^{k} s_{i}\left(t_{i+1}\right) / d t^{k}=d^{k} s_{i+1}\left(t_{i+1}\right) / d t^{k}
$$

for $k=0,1, \ldots, 2 q-2$ and $i=1, \ldots, N-2$, where we denote by $s_{i}(t)$ the restriction of $s(t)$ to the interval $\left(t_{i}, t_{i+1}\right)$.

Conditions (i)-(iii) together with the interpolation property (1.2) may be considered as an alternative definition of splines which, chronologically, is the original; cf. [ANW, p. 76], [Laur, p. 162].

In the multivariate case, a natural analog to problem (1.1) is the following one:

$$
\int_{\bar{D}}\left(\Delta^{q} f(x)\right)^{2} d x \rightarrow \inf ,
$$

where the minimization is over a proper class of functions, $D$ is a bounded domain in $\mathbf{R}^{n}$, and $\Delta^{q}$ is the $q$ times iterated Laplace operator.

The purpose of the present paper is to provide a proper setting for the solubility of problem (1.3). In other words, we find an analog to the interpolation conditions (1.2) for which problem (1.3) has a solution.

As a result we obtain a constructive solution to problem (1.3) and an analog to conditions (i)-(iii).

In fact, the set of $N$ points in $[a, b]$ is replaced by a set of $N$ closed smooth surfaces in $\bar{D}$, namely $T_{1}, T_{2}, \ldots, T_{N}$, such that $T_{j}$ surrounds $T_{j-1}$ for $j=1,2, \ldots, N$, and $T_{N}=\partial D$; it is convinient to put $T_{0}=\emptyset$. Condition (1.2) is replaced by

$$
f(x)=g(x)
$$

for $x \in S T=\bigcup_{j=1}^{N} T_{j}$, where $g(x)$ is a function defined on all surfaces $T_{j}, j=$ $1,2, \ldots, N$, and $\left(1.2^{\prime}\right)$ is replaced by the following condition:

$$
\begin{gathered}
\Delta^{k} f(x)=0, \quad x \in T_{N}=\partial D, \text { for } k=1, \ldots, q-1 ; \\
\left(\partial / \partial n_{N}\right) \Delta^{k} f(x)=0, \quad x \in T_{N}=\partial D, \text { for } k=0, \ldots, q-1 ;
\end{gathered}
$$

here $n_{N}$ denotes the inner unit normal vector to $T_{N}$. 
The solution $s(x)$ to problem (1.3)-(1.4') will be called a polyspline of order $2 q$. In case it exists, it satisfies the following analogs to conditions (i)-(iii) (for the proof see Theorem 4.2 below):

(i') It is a $2 q$-harmonic function in the complement of the surfaces $T_{j}$, i.e.

$$
\Delta^{2 q} s(x)=0, \quad x \in D \backslash S T .
$$

(ii') On the boundary $\partial D$ the following condition holds:

$$
\begin{gathered}
\Delta^{k} s(x)=0, \quad x \in \partial D=T_{N}, \quad \text { for } k=1, \ldots, q-1 \\
\left(\partial / \partial n_{N}\right) \Delta^{k} s(x)=0, \quad x \in \partial D=T_{N}, \quad \text { for } k=0, \ldots, q-1 .
\end{gathered}
$$

(iii') If we denote by $s_{j}(x)$ the restriction of $s(x)$ to the layer lying between $T_{j}$ and $T_{j-1}$, then

$$
\Delta^{p} s_{j+1}(x)=\Delta^{p} s_{j}(x), \quad x \in T_{j},
$$

for $p=0,1, \ldots, 2 q-1$ and $j=1,2, \ldots, N-1$;

$$
\left(\partial / \partial n_{j}\right) \Delta^{p} s_{j+1}(x)=\left(\partial / \partial n_{j}\right) \Delta^{p} s_{j}(x), \quad x \in T_{j},
$$

for $p=0,1, \ldots, 2 q-2$, and $j=1,2, \ldots, N-1$. Here $\left(\partial / \partial n_{j}\right)$ denotes the inner normal derivative on the surface $T_{j}$.

Now, conditions $\left(\mathrm{i}^{\prime}\right)-\left(\mathrm{iii}^{\prime}\right)$ may be taken as a definition of a polyspline of order $2 q$.

In the simplest case, $q=1$, we have pieces of biharmonic functions which are matching on the common boundaries $T_{j}$ of the layers, in the sense that $s_{j+1}(x)=$ $s_{j}(x), \partial s_{j+1}(x) / \partial n_{j}=\partial s_{j}(x) / \partial n_{j}$ and $\Delta s_{j+1}(x)=\Delta s_{j}(x)$ for all $x \in T_{j}$ and $j=1,2, \ldots, N-1$; on the boundary $\partial D=T_{N}$ we have $\left(\partial / \partial n_{N}\right) s_{N}(x)=0$.

The following argument shows the roots of the analogy with one-dimensional spline theory (see [ANW, p. 109]):

Let us check that the number of degrees of freedom (or "free parameters") is equal to the number of matching conditions plus the number of interpolation conditions. According to the theory of higher order elliptic boundary value problems [ADN], [LiMa], $[\mathrm{H}]$, for the function $u_{j}$ polyharmonic of order $2 q$, on every surface $T_{j}, j=1, \ldots, N-1$, we have $2 q$ boundary conditions (playing the role of degrees of freedom), and similarly $2 q$ for $u_{j+1}$. The number of constraints (ii') and (iii') is $4 q-1$. One degree of freedom is necessary for the data interpolation (1.4) on $T_{j}$ . On the surface $T_{N}$ we have $2 q$ boundary conditions for the function $u_{N}$. The number of constraints (ii') is $2 q-1$. One degree of freedom is necessary for the data interpolation (1.4) on $T_{N}$. Consequently, the number of degrees of freedom is equal to the number of matching conditions in $\left(\mathrm{i}^{\prime}\right)-\left(\mathrm{iii}{ }^{\prime}\right)$ plus the number of the interpolation conditions (1.4).

a) The following are briefly the main results of the paper:

In the Sobolev spaces $H^{s}$ we prove existence for polysplines of order $2 q$ satisfying the interpolation conditions, Theorem 6.3 .1 below. Let us remark that with the same success one may study the existence in the Hölder spaces $C^{k+\alpha}$ following the approach in $[\mathrm{ADN}]$.

The core of the paper is a basic identity for polysplines which is analogous to the fundamental identity for one-dimensional splines [ANW, Laur]. By virtue of this identity we obtain uniqueness for interpolation polysplines, Theorem 4.1, and the equivalence of the two definitions, Theorem 4.2, the one given through problem $(1.3)-\left(1.4^{\prime}\right)$, and the other through properties $\left(\mathrm{i}^{\prime}\right)-\left(\mathrm{iii}^{\prime}\right)$ and $(1.4)-\left(1.4^{\prime}\right)$. That is 
the analog to the well-known extremal property of one-dimensional splines, called Holladay's theorem [ANW, Theorems 3.1.1, 5.4.1], [Laur, p. 162].

b) The following should be said about the techniques of the proofs:

In one-dimensional spline theory uniqueness implies existence of splines. Here due to the basic identity of Theorem 3.1 we obtain the same principle: uniqueness implies existence of polysplines through the Fredholm alternative proved in Theorem 6.2.4. Let us remark the exceptional role of the order $2 q$. For solutions of the equation $\Delta^{2 q+1} u=0$ one cannot obtain the key identity of Theorem 3.1, and so the uniqueness of the interpolation $2 q+1$-polysplines does not hold in general and Corollary 6.2.3 fails. That is rather similar to the one-dimensional case where the splines of odd order (solutions to $d^{2 k} u / d t^{2 k}=0$ ) satisfy a basic identity and uniqueness of interpolation, while the even order splines do not enjoy similar properties.

We provide the existence theorem for an arbitrary integer $q \geq 1$. The most technical aspect of this is Proposition 5.1.1, the proof of which is reduced to checking the condition of Agmon-Douglis-Nirenberg [ADN], [LiMa, Section 2.4.1], [Nec] for a special system of boundary operators.

c) The plan of the paper is the following:

In Section 2 we provide some basic notions and notations.

In Section 3 we prove the basic identity for polysplines.

In Section 4 we prove the uniqueness for interpolation polysplines.

A reader who is not experienced in a priori estimates for higher order elliptic equations, or is interested in the subject mainly from the point of view of splinetheory, may drop Sections 5, 6. The existence Theorem 6.3.1 will be enough for him. Still we would encourage the reader to go especially through Section 5.1 to make sure that modulo some rather standard techniques of frozen coefficients things are essentially reduced to one-dimensional generalized interpolation splines, or, even more $L$-splines (cf. [ANW], [Schum, Chapter 10] for the notions of generalized and $L$-splines); see Proposition 5.1.1 below. This point of view is strongly supported by the examples in Section 7, where in the case of data sets with symmetry the effective computation of interpolation polysplines is reduced through Fourier or Mellin transform to finding a one-dimensional interpolation $L$-spline. In fact, the main result of Section 5, Theorem 5.2.1, is essentially identical with a detailed consideration of one of the important special cases of data sets $S T$ possessing symmetry. That is the case of parallel hyperplanes outlined in Section 7,2 ).

For someone familiar with the a priori estimates for higher order elliptic equations we have to say that in Sections 5,6 we follow closely the scheme for proving existence for boundary value problems presented in [LiMa], $[\mathrm{H}]$. Trying to make the presentation brief but clear, we write the chain of theorems leading to the existence result, Theorem 6.3.1, but omit the proofs which are similar to those in [LiMa], or indicate only the novelty which arises.

In Section 5 we consider a more general problem for interpolation polysplines than is necessary, namely, when the right sides are non-homogeneous. In a forthcoming paper this more general result will be used for proving existence of "smoothing" and "perfect" polysplines.

In Section 6 we provide a priori estimates in an arbitrary domain with interior boundary $\bigcup_{j=1}^{N} T_{j}$. The existence is proved in the general form in Section 6.2. The final existence result for polysplines is Theorem 6.3.1. 
d) Let us remark that biharmonic functions and solutions of other higher order elliptic equations find wide applications in fitting discrete scattered data. In this direction the following works are known to the author: solutions of higher order elliptic equations for interpolating and smoothing purposes are studied in [FJ], [Du]. An algorithm was given by Briggs in [Bri] (see also the papers [GA], [Smi]). Polyharmonic functions are used for discrete data fitting in [Ma], [Wa].

e) Some of the results of the present paper were announced in [Ko1], [Ko2] in a more general form. The paper itself is an extended version of the preprint [Ko5].

f) We number the formulas, theorems, propositions, and lemmas within every subsection.

After having introduced the notion of polyspline by a direct analogy with the univariate case, it is natural to ask how far does the analogy extend. Let us indicate some problems that seem to be interesting and unsolved:

1. From the point of view of applications it is natural to have a data set $S T$ which is a union of intersecting smooth manifolds, i.e., when $S T$ itself is a manifold with singularities. In $\mathbf{R}^{2}$ in the case of such a data set $S T$ polysplines are studied in [Ko3]. So far, in dimensions greater than 2 there is no complete theory of elliptic boundary value problems in domains with singularities, and, for that reason, the results of the paper are not extendable at the present moment.

2. It would be interesting to have polysplines with a compact support, i.e. analogs to the one-dimensional B-splines. In particular, a B-polyspline in the biharmonic case will satisfy $u=0, \partial u / \partial n=0, \Delta u=0$ on $\partial D$. Assuming $\partial D=T_{N}$ given, the existence and smoothness of the rest of the components $T_{j}$ of the manifold $S T$ seems to be an uneasy free boundary value problem. At least one case of nontrivial B-polyspline is described in [HK] when the set $S T$ consists of $q$ concentric spheres (it is also a piecewise polyharmonic spline in the sense of [Ma]). There it arises as a Peano kernel of a mean value property of Bramble-Payne for polyharmonic functions, showing a beautiful analogy with the univariate $B$-splines. The last arise like Peano kernels of the divided difference operator; cf. [Schum, Chapter 4.3, also p. 182].

\section{Preliminaries}

a) Let $D$ be a bounded domain in $\mathbf{R}^{n}$ with infinitely smooth boundary $\Gamma$.

Suppose that a family of subdomains $\tilde{D}_{1}, \ldots, \tilde{D}_{N}, \tilde{D}_{N}=D$, is given such that the closure $\operatorname{cl}\left(\tilde{D}_{j}\right)$ lies in the domain $\tilde{D}_{j+1}$, i.e. $\operatorname{cl}\left(\tilde{D}_{j}\right) \subseteq \tilde{D}_{j+1}, j=1,2, \ldots, N-1$.

We shall denote by $T_{j}$ the boundary of $\tilde{D}_{j}, T_{j}=\partial\left(\tilde{D}_{j}\right), j=1,2, \ldots, N$, and so we have $T_{N}=\partial D$; for convenience we put $T_{0}=\emptyset$.

By $D_{j}$ we shall denote the layer which lies between the surfaces $T_{j}$ and $T_{j+1}$, i.e.

$$
D_{j}=\tilde{D}_{j} \backslash \operatorname{cl}\left(\tilde{D}_{j-1}\right) \quad \text { for } j=2, \ldots, N
$$

we put $D_{1}=\tilde{D}_{1}$.

By $n_{j}$ we shall denote the inner unit normal vector to $T_{j}$.

The union of the surfaces

$$
S T=\bigcup_{j=1}^{N} T_{j}
$$

is considered to be the set of data points (parameter set) for the polysplines. 
We shall suppose throughout the paper that the boundaries $T_{j}, j=1,2, \ldots, N$, are infinitely smooth surfaces of dimension $n-1$, which are conditions providing solubility of the boundary elliptic problems and the validity of Green's formulas [Aron], [LiMa]. The proof of the existence theorem based on the techniques of a priori estimates is the main reason for these severe smoothness requirements on the boundaries [LiMa, p. 111], [H, Chapter XX], although these conditions may be essentially relaxed.

The simplest model example to consider, is the one where $D$ is a ball in $\mathbf{R}^{n}$ and $\tilde{D}_{j}, j=1,2, \ldots, N$, are $N$ concentric balls. Then $D_{j}, j=2, \ldots, N$, is the spherical layer between the spheres $T_{j-1}=\partial \tilde{D}_{j-1}$ and $T_{j}=\partial \tilde{D}_{j}$; we have $D_{1}=\tilde{D}_{1}$.

b) In what follows we shall use the standard notations and results concerning spaces of functions on domains and their boundaries, which can be found in [LiMa], [Nec].

We shall denote by $H^{s}(\Omega)$ the Sobolev space for a domain $\Omega \subseteq \mathbf{R}^{n}$, and by $H^{s}(\Gamma)$ the Sobolev space for the boundary $\Gamma=\partial \Omega$.

For a compact domain $\Omega$, we shall denote by $C^{\infty}(\Omega)$ the space of infinitely differentiable functions on $\Omega$. We denote by $\mathcal{D}(\Omega)$ the space of infinitely differentiable functions with supports in $\Omega$.

c) We shall denote by $\Delta$ the Laplace operator in $\mathbf{R}^{n}$ given by

$$
\sum_{j=1}^{n} \partial^{2} / \partial x_{j}^{2}
$$

and by $\Delta^{k}$ the polyharmonic operator, which is defined inductively for all integers $k \geq 0$ by $\Delta^{0}=1, \Delta^{1}=\Delta$ and $\Delta^{k}=\Delta \Delta^{k-1}$.

The space $H^{s}(D \backslash S T)$ should be understood as the space of functions $f$ for which $f \in H^{s}\left(D_{j}\right)$ for every connected component $D_{j}$ of $D \backslash S T$.

It is important to introduce a proper space of functions which arises naturally in Theorems 3.1 and 4.2 below. Namely, we will denote by $H L_{q}$ the space of functions $f \in H^{2 q}(D \backslash S T)$ such that the traces of the functions $\Delta^{k} f$ and $\left(\partial / \partial n_{j}\right) \Delta^{k} f$, for every $k=0,1, \ldots, q-1$, coincide on $T_{j}$ when taken from $D_{j}$ and from $D_{j+1}$.

\section{Interpolation Polysplines - Definition and Basic Identity}

For a function $u(x)$ defined in the domain $D$ we shall denote by $u_{j}(x)$ its restrictions to the subdomains $D_{j}, j=1,2, \ldots, N$.

Definition. Let $q>0$ be a given integer. The function $u \in H^{4 q}(D \backslash S T)$ is called an (interpolation) polyspline of order $2 q$ (for the given subdivision by the domains $\tilde{D}_{j}$ ) if and only if the following conditions hold:

$$
\begin{gathered}
\Delta^{2 q} u_{j}(x)=0, \quad x \in D_{j}, \quad \text { for } j=1,2, \ldots, N ; \\
\Delta^{k} u_{N}(x)=0, \quad x \in T_{N}=\partial D, \quad \text { for } k=1, \ldots, q-1 ; \\
\left(\partial / \partial n_{N}\right) \Delta^{k} u_{N}(x)=0, \quad x \in T_{N}=\partial D, \quad \text { for } k=0, \ldots, q-1 ; \\
\Delta^{p} u_{j}(x)=\Delta^{p} u_{j+1}(x), \quad x \in T_{j},
\end{gathered}
$$

for $j=1,2, \ldots, N-1$ and $p=0,1, \ldots, 2 q-1$; 


$$
\left(\partial / \partial n_{j}\right) \Delta^{p} u_{j}(x)=\left(\partial / \partial n_{j}\right) \Delta^{p} u_{j+1}(x), \quad x \in T_{j},
$$

for $j=1,2, \ldots, N-1$ and $p=0,1, \ldots, 2 q-2$.

Equalities (3.2)-(3.4) should be considered as equalities between traces of functions.

The space of all such polysplines will be denoted by $P S_{2 q}(D)$, or simply by PS.

The following is a basic identity for polysplines:

Theorem 3.1. For every $h \in H L_{q}(D)$ and $u \in P S_{2 q}(D)$ the following equality is true:

$$
\begin{gathered}
\int_{D} \Delta^{q} u(x) \Delta^{q} h(x) d x=\int_{S T \backslash \partial D}\left\{(\partial / \partial n) \Delta^{2 q-1} u(x)\right\}_{j u m p} h(x) d \omega(x) \\
-\sum_{l=0}^{q-1} \int_{T_{N}}\left\{\Delta^{l+q} u(x)\left(\partial / \partial n_{N}\right) \Delta^{q-1-l} h(x)\right. \\
\left.-\left(\partial / \partial n_{N}\right) \Delta^{l+q} u(x) \Delta^{q-1-l} h(x)\right\} d \omega_{N}(x),
\end{gathered}
$$

where $\left\{(\partial / \partial n) \Delta^{2 q-1} u(x)\right\}_{\text {jump }}$ denotes the jump of the normal derivative at the point $x \in T_{j}$, which is equal to

$$
\left(\partial / \partial n_{j}\right) \Delta^{2 q-1} u_{j}(x)-\left(\partial / \partial n_{j}\right) \Delta^{2 q-1} u_{j+1}(x)
$$

for $j=1, \ldots, N-1$. (For $j=N$ we have only one term, $\left(\partial / \partial n_{N}\right) \Delta^{2 q-1} u_{N}(x)$.) Here $\omega(x)$ denotes the intrinsic measure on all $T_{j}, j=1, \ldots, N$.

Proof. By the Green's formula in [Aron, p. 10, (2.9)], for every $j$ we have

$$
\begin{gathered}
\int_{D_{j}} \Delta^{q} u(x) \Delta^{q} h(x) d x+\sum_{l=0}^{q-1} \int_{\partial D_{j}}\left\{\Delta^{l+q} u(x)\left(\partial / \partial \nu_{j}\right) \Delta^{q-1-l} h(x)\right. \\
\left.-\left(\partial / \partial \nu_{j}\right) \Delta^{l+q} u(x) \Delta^{q-1-l} h(x)\right\} d \sigma_{j}(x)=0,
\end{gathered}
$$

where $d \sigma_{j}(x)$ denotes the element of the intrinsic measure on $\partial D_{j}$, and $\nu_{j}$ is the inner unit normal vector to $D_{j}$.

Taking into account that $\nu_{j}=n_{j}$ on $T_{j}$ and $\nu_{j}=-n_{j-1}$ on $T_{j-1}$, after summing over $j=1, \ldots, N$, we obtain formula (3.6).

Q.E.D

\section{Uniqueness of Interpolation Polysplines and Extremal Property}

The immediate profit of Theorem 3.1 is the uniqueness of polysplines.

Theorem 4.1. Let $u(x)$ be an interpolation polyspline of order $2 q$. If $u(x)=0$ on the set $S T$, then $u \equiv 0$ in the whole domain $D$.

Proof. Let us put $h(x)=u(x)$ in formula (3.6) of Theorem 3.1. We obtain

$$
\int_{\bar{D}}\left(\Delta^{q} u(x)\right)^{2} d x=0
$$

This implies that $\Delta^{q} u_{j}(x)=0$, for every $x \in \bar{D}_{j}, j=1, \ldots, N$. 
From Lemma 4.1 below it follows that $u(x)$ is a $q$-harmonic function everywhere in $D$. Now the boundary conditions (3.2) imply that $u \equiv 0$ in $D$.

The following is in fact a continuation through the boundary result:

Lemma 4.1. Let the function $u \in H^{2 m}(D \backslash S T)$ have the property that $\Delta^{m} u(x)=0$ for every $D_{j}, j=1, \ldots, N$, and also satisfy the following boundary conditions:

$$
\text { (i) } \Delta^{p} u_{j}(x)=\Delta^{p} u_{j+1}(x)
$$

$$
\text { (ii) }\left(\partial / \partial n_{j}\right) \Delta^{p} u_{j}(x)=\left(\partial / \partial n_{j}\right) \Delta^{p} u_{j+1}(x)
$$

for every $x \in T_{j}$, where $p=0,1, \ldots, m-1$ and $j=1, \ldots, N-1$. Then $\Delta^{m} u(x)=0$ for every $x \in D$.

Proof. Let us apply the second Green's formula [Aron, p. 10, (2.11)] to every subdomain $D_{j}$. We obtain

$$
\begin{gathered}
\Omega_{n} \sum_{l=0}^{m-1} \int_{\partial D_{j}}\left\{\Delta^{l} u(x)\left(\partial / \partial \nu_{j}\right) r_{l+1}(x-y)\right. \\
\left.-\left(\partial / \partial \nu_{j}\right) \Delta^{l} u(x) r_{l+1}(x-y)\right\} d \omega_{j}(x) \\
-\Omega_{n} \int_{D_{j}} \Delta^{m} u(x) r_{m}(x-y) d y=u(y), \text { for } y \in D_{j}
\end{gathered}
$$

and is equal to 0 elsewhere. Here $\Omega_{n}$ is a suitable constant and $r_{l}(x)$ is a normalized fundamental solution of the equation $\Delta^{l} w(x)=0$ [Aron, pp. 8-10].

Since $\nu_{j}=-\nu_{j+1}$ on $T_{j}$, after summing over $j$ in (4.1), we obtain the second Green's formula for $u(y)$ in $D$, which proves that it is a polyharmonic function of order $m$ in the whole of $D$.

Q.E.D

Now we may prove that the solution to problem (3.1)- (3.4) satisfying the interpolation conditions (1.4) is in fact a solution to the variational problem (1.3)-(1.4').

Theorem 4.2. The solution $v(x)$ to problem (3.1)-(3.4) satisfying (1.4) is also a solution to the following extremal problem:

$$
\int_{D}\left[\Delta^{q} f(x)\right]^{2} d x \rightarrow \inf ,
$$

where the infimum is taken over the set of functions $f(x) \in H^{2 q}(D)$ satisfying conditions (1.4) $-\left(1.4^{\prime}\right)$

Proof. For an arbitrary $f$ consider the difference

$$
\begin{aligned}
& \int_{D}\left[\Delta^{q} f(x)-\Delta^{q} v(x)\right]^{2} d x \\
& \quad=\int_{D}\left[\Delta^{q} f(x)\right]^{2} d x-2 \int_{D}\left[\Delta^{q} f(x)-\Delta^{q} v(x)\right] \Delta^{q} v(x) d x-\int_{D}\left[\Delta^{q} v(x)\right]^{2} d x .
\end{aligned}
$$

Applying Theorem 3.1 to the second term by putting $h=v-f$ and $u=v$, we obtain the equality 


$$
\begin{gathered}
\int_{D}\left[\Delta^{q} f(x)-\Delta^{q} v(x)\right] \cdot \Delta^{q} v(x) d x \\
=\int_{S T \backslash \partial D}[f(x)-v(x)] \cdot\left\{(\partial / \partial n) \Delta^{2 q-1} v(x)\right\} d \omega(x) \\
-\sum_{l=0}^{q-1} \int_{T_{N}}\left\{\Delta^{l+q} v(x)\left(\partial / \partial n_{N}\right) \Delta^{q-1-l}(f(x)-v(x))\right. \\
\left.-\left(\partial / \partial n_{N}\right) \Delta^{l+q} v(x) \Delta^{q-1-l}(f(x)-v(x))\right\} d \omega_{N}(x) .
\end{gathered}
$$

All terms on the right side are zero, since $v$ and $f$ satisfy $(1.4)-\left(1.4^{\prime}\right)$. Thus we obtain

$$
\int_{D}\left[\Delta^{q} v(x)\right]^{2} d x=\int_{D}\left[\Delta^{q} f(x)\right]^{2} d x-\int_{D}\left[\Delta^{q} f(x)-\Delta^{q} v(x)\right]^{2} d x,
$$

which proves our statement.

\section{A Priori Estimates for Interpolation Polysplines IN THE HALF-SPACE}

We will call the function $g(x)$ defined on the set $S T$ interpolation data for the (interpolation) polyspline $u(x) \in P S_{2 q}$ if and only if

$$
u=g \quad \text { on } S T
$$

for the trace of the function $u(x)$ on the set $S T$.

Our goal is a priori estimates for interpolation polysplines of the type

$$
\|u\|_{H^{s+4 q}(D \backslash S T)} \leq C\left\{\|g\|_{H^{s+4 q-1 / 2}(S T)}+\|u\|_{H^{s+4 q-1}(D \backslash S T)}\right\} .
$$

They are proved for the case of non-homogeneous interior boundary conditions in Theorem 5.2.1 below.

5.1. Basic Proposition for the Matching in the Half-spaces. We will first prove the main technical result of the paper, Proposition 5.1.1, necessary for studying the a priori estimates for operators with constant coefficients in the half-space.

For this purpose we recall the standard notations and notions from [LiMa].

We will use the notation $x=(y, t)$ for a point $x \in \mathbf{R}^{n}$, where $y=\left(y_{1}, \ldots, y_{n-1}\right) \in$ $\mathbf{R}^{n-1}, t \in \mathbf{R}$. The variable dual to $x$ will be $\xi=\left(\eta, t^{\prime}\right)$, where $\eta=\left(\eta, \ldots, \eta_{n-1}\right) \in$ $\mathbf{R}^{n-1}$ and $t^{\prime} \in \mathbf{R}$.

We denote by $\mathbf{R}_{+}^{n}$ the half-space of the points $x \in \mathbf{R}^{n}$ with $t>0$; by $\mathbf{R}_{-}^{n}$ we denote the other half-space, where $t<0$.

Let us put $m=2 q$, and consider the operators

$$
\begin{gathered}
A(D) u=A\left(D_{y}, D_{t}\right) u=\Delta^{m} u, \\
B_{j}(D) u=B_{j}\left(D_{y}, D_{t}\right) u=\Delta^{j} u, \quad j=0, \ldots, m-1, \\
S_{j}(D) u=S_{j}\left(D_{y}, D_{t}\right) u=D_{t} \Delta^{j} u, \quad j=0, \ldots, m-1,
\end{gathered}
$$

where $D_{y}$ denotes the multivariate differentiation in $y$ and $D_{t}=\partial / \partial t$.

We will put $m_{j}=2 j=\operatorname{ord}\left(B_{j}\right)$. Hence the order of the operator $S_{j}$ is $m_{j}+1$, for $j=0, \ldots, m-1$. 
Let us denote by $\tau_{i}^{+}(\eta)$ (respectively by $\tau_{i}^{-}(\eta)$ ) the roots of $A(\eta, \tau)$ with positive (resp. negative) imaginary part. Since $A(D)$ has real coefficients, we may write that $\tau_{j}^{-}(\eta)=\overline{\tau_{j}^{+}(\eta)}, j=1, \ldots, m$, where the bar means a complex conjugate.

We denote by $S\left(\mathbf{R}_{+}\right)$, as usual (cf. [Schw]), the space of functions which are infinitely differentiable for $t \geq 0$ and are rapidly decreasing for $t \rightarrow \infty$ (i.e. $t^{k} \phi^{(j)}(t) \rightarrow 0$ as $t \rightarrow \infty$, for every $k$ and every $j$ ).

We denote by $S\left(\mathbf{R}_{-}\right)$the similar space for $t \leq 0$, i.e. $\phi(t) \in S\left(\mathbf{R}_{-}\right)$if and only if $\phi(-t) \in S\left(\mathbf{R}_{+}\right)$.

For every $\eta \in \mathbf{R}^{n-1}, \eta \neq 0$, let us consider the system of ordinary differential equations

$$
\begin{aligned}
& A\left(\eta, i^{-1} d / d t\right) \phi(\eta, t)=0, \quad t \geq 0, \\
& A\left(\eta, i^{-1} d / d t\right) \psi(\eta, t)=0, \quad t \leq 0,
\end{aligned}
$$

with boundary conditions

$$
B_{j}\left(\eta, i^{-1} d / d t\right) \phi(\eta, t)_{\mid t=0}=B_{j}\left(\eta, i^{-1} d / d t\right) \psi(\eta, t)_{\mid t=0}+c_{j},
$$

for $j=0, \ldots, m-1$, and

$$
S_{j}\left(\eta, i^{-1} d / d t\right) \phi(\eta, t)_{\mid t=0}=S_{j}\left(\eta, i^{-1} d / d t\right) \psi(\eta, t)_{\mid t=0}+d_{j},
$$

for $j=0, \ldots, m-2$;

$$
B_{0}\left(\eta, i^{-1} d / d t\right) \phi(\eta, t)_{\mid t=0}=\gamma,
$$

where $\phi(\eta, t) \in S\left(\mathbf{R}_{+}\right), \psi(\eta, t) \in S\left(\mathbf{R}_{-}\right)$, and $c_{j}, d_{j}, \gamma$ are given complex numbers.

The following statement is an analog to Proposition 4.2, Ch. 2 in [LiMa].

Proposition 5.1.1. Let the operators $A_{2 q}$ and $B_{j}, j=0, \ldots, 2 q-1$, and $S_{j}, j=$ $0, \ldots, 2 q-2$, be given by (5.1.1)-(5.1.3). Then problem (5.1.4)-(5.1.8) has a unique solution for every set of constants $c_{j}, j=0, \ldots, m-1$, and $d_{j}, j=0, \ldots, m-2$, and $\gamma$.

Proof. a) Let us find the dimension of the space of solutions to the system (5.1.4)(5.1.5).

Let us recall the simple fact from the theory of ordinary differential equations that the solutions to the equation (5.1.4) (or to (5.1.5)) are exponential polynomials. An exponential polynomial in $\mathbf{R}_{+}$is in $S\left(\mathbf{R}_{+}\right)$if and only if the exponents have positive imaginary parts. Similarly, an exponential polynomial in $\mathbf{R}_{-}$is in $S\left(\mathbf{R}_{-}\right)$ if and only if the exponents have negative imaginary parts. So if we denote by

$$
\tau^{+}=i|\eta| \text { and } \tau^{-}=-i|\eta|
$$

the zeroes of the polynomial equation $A_{m}(\eta, \tau)=\left(\eta^{2}+\tau^{2}\right)^{m}=0$ (which clearly have multiplicity $m=2 q$ ), we obtain that the exponential polynomials in $S\left(\mathbf{R}_{+}\right)$ contain exponents of the kind $e^{i t \tau^{+}}$, and exponential polynomials in $S\left(\mathbf{R}_{-}\right)$contain exponents of the kind $e^{i t \tau^{-}}$. Since the multiplicity of $\tau^{+}$is $m$, the space of solutions to equation (5.1.4) has complex dimension $m$. For the same reasons, the dimension of the space of solutions to equation (5.1.5) also has complex dimension $m$. Consequently, the space of solutions to the system (5.1.4)-(5.1.5) has complex dimension $2 m$. 
b) An important observation for the further considerations is that if an exponential polynomial $\phi(\eta, t) \in S\left(\mathbf{R}_{+}\right)$is a solution to equation (5.1.4), then the function $\phi(\eta,-t) \in S\left(\mathbf{R}_{-}\right)$is a solution to equation (5.1.5), i.e.

$$
A_{m}\left(\eta, i^{-1} d / d t\right) \psi(\eta,-t)=0 .
$$

The last is due to the fact that the polynomial $A_{m}(\eta, \tau)$ depends on $\tau^{2}$ solely.

c) As proved above, the dimension of the space of solutions to equations (5.1.4) (5.1.5) is exactly $2 m$, which is equal to the number of free parameters on the right-hand side of the boundary conditions (5.1.6)-(5.1.8), namely the constants $c_{j}, j=0, \ldots, m-1 ; d_{j}, j=0, \ldots, m-2$, and $\gamma$.

Thus to every couple $(\phi, \psi) \in S\left(\mathbf{R}_{+}\right) \times S\left(\mathbf{R}_{-}\right)$which is a solution to the system (5.1.4)-(5.1.5), we map an element of the space $\mathbf{C}^{2 m}$, given by the following sequence of $2 m$ numbers:

$$
B_{j}\left(\eta, i^{-1} d / d t\right) \phi(\eta, t)_{\mid t=0}-B_{j}\left(\eta, i^{-1} d / d t\right) \psi(\eta, t)_{\mid t=0},
$$

for $j=0, \ldots, m-1$;

$$
S_{j}\left(\eta, i^{-1} d / d t\right) \phi(\eta, t)_{\mid t=0}-S_{j}\left(\eta, i^{-1} d / d t\right) \psi(\eta, t)_{\mid t=0},
$$

for $j=0, \ldots, m-2$;

$$
B_{0}\left(\eta, i^{-1} d / d t\right) \phi(\eta, t)_{\mid t=0} .
$$

This is in fact a map from a $2 m$-dimensional linear space into $\mathbf{C}^{2 m}$. Consequently, the system (5.1.4)-(5.1.8) is solvable if and only if for a zero right-hand side,

$$
c_{j}=0, j=0, \ldots, m-1 ; d_{j}=0, j=0, \ldots, m-2 ; \gamma=0,
$$

we have that $\phi=0, \psi=0$.

Suppose that (5.1.12) holds true.

Since the operators $B_{j}, j=0, \ldots, m-1$, depend on $\tau^{2}$ solely, it follows that the solution to equation (5.1.5) satisfies

$$
B_{j}\left(\eta, i^{-1} d / d t\right) \psi(\eta,-t)_{\mid t=0}=B_{j}\left(\eta, i^{-1} d / d t\right) \phi(\eta, t)_{\mid t=0},
$$

for $j=0, \ldots, m-1$.

In view of $(5.1 .10)$ the functions $\psi(\eta,-t)$ and $\phi(\eta, t)$ are solutions to the same boundary value problem. Now we may apply Proposition 4.2 , Ch.2 in [LiMa] to the operators $A_{m}$ and $B_{j}, j=0, \ldots, m-1$ (since they satisfy hypothesis II there), which gives uniqueness of the solution (we provide this result in Lemma 5.1.2,i) below).

Hence, we obtain that

$$
\phi(\eta, t)=\psi(\eta,-t) .
$$

On the other hand, notice that the operators $S_{j}\left(\eta, i^{-1} d / d t\right)$ are given by

$$
S_{j}\left(\eta, i^{-1} d / d t\right)=i^{-1} d / d t\left\{B_{j}\left(\eta, i^{-1} d / d t\right)\right\}, \quad j=0, \ldots, m-2 .
$$

Consequently,

$$
S_{j}\left(\eta, i^{-1} d / d t\right) \psi(\eta,-t)_{\mid t=0}=-S_{j}\left(\eta, i^{-1} d / d t\right) \psi(\eta, t)_{\mid t=0},
$$

for $j=0, \ldots, m-2$.

By virtue of (5.1.7) and (5.1.14) we obtain the following equalities:

$$
S_{j}\left(\eta, i^{-1} d / d t\right) \psi(\eta, t)_{\mid t=0}=0, \quad j=0, \ldots, m-2 .
$$


The boundary condition (5.1.8) is

$$
B_{0}\left(\eta, i^{-1} d / d t\right) \phi(\eta, t)_{\mid t=0}=0 .
$$

d) The last step is to see that $\phi \equiv \psi \equiv 0$.

For proving it we will use an idea of spline theory where the uniqueness (and existence) of the splines is proved through a suitable identity (cf. [ANW, p. 154]) (which we have already exploited in Section 4).

For the function $w(\eta, t)$ which coincides with $\phi$ on $\mathbf{R}_{+}$and with $\psi$ on $\mathbf{R}_{-}$we have the following identity:

$$
\begin{aligned}
\int_{-\infty}^{\infty} A_{q}\left(\eta, i^{-1} d / d t\right) w(\eta, t) A_{q}\left(\eta, i^{-1} d / d t\right) f(t) d t \\
=\left\{S_{2 q-1} w(0)\right\} f(0)
\end{aligned}
$$

for every $f \in H^{2 q}(\mathbf{R})$; here we use the notation for the jump:

$$
\left\{S_{2 q-1} w(0)\right\}=S_{2 q-1}\left(\eta, i^{-1} d / d t\right) \phi(\eta, t)_{\mid t=0}-S_{2 q-1}\left(\eta, i^{-1} d / d t\right) \psi(\eta, t)_{\mid t=0} .
$$

The proof of (5.1.18), like that of Theorem 3.1, consists of simple integration by parts, and makes use of the boundary conditions (5.1.13) and (5.1.15), and the fact that

$$
A_{2 q}\left(\eta, i^{-1} d / d t\right) w(\eta, t)=0 \text { for every } t \neq 0 .
$$

Let us put $f=w$ in (5.1.18). Since $B_{0} \phi_{\mid t=0}=B_{0} \psi_{\mid t=0}=w(0)=0$, we obtain

$$
\int_{-\infty}^{\infty}\left[A_{q}\left(\eta, i^{-1} d / d t\right) w(\eta, t)\right]^{2} d t=0
$$

and, consequently,

$$
A_{q}\left(\eta, i^{-1} d / d t\right) w(\eta, t)=0
$$

for every $t \in \mathbf{R}$.

Further we use (5.1.19) and also (5.1.15)-(5.1.16) to obtain the following boundary value problem:

$$
\begin{gathered}
A_{q}\left(\eta, i^{-1} d / d t\right) \phi(\eta, t)=0, \quad t \geq 0 \\
S_{j}\left(\eta, i^{-1} d / d t\right) \phi(\eta, t)_{\mid t=0}=0, \quad j=0,1, \ldots, q-1 .
\end{gathered}
$$

This problem has only the trivial solution, since the operators $A_{q}$ and $S_{j}, j=$ $0,1, \ldots, q-1$, satisfy hypothesis II of Ch. 2 in [LiMa], (and we may apply Proposition 4.2, Ch. 2, same ref.); see also Lemma 5.1.2,ii) below.

The last implies that $\phi \equiv 0$.

The proof of the proposition is finished.

For completeness we provide the following (cf. [Nec]; B.V.P. means "boundary value problem"):

Lemma 5.1.2. i) The boundary value problem (5.1.4) with

$$
B_{j}\left(\eta, i^{-1} d / d t\right) \phi(\eta, t)_{\mid t=0}=0, \quad j=0, \ldots, m-1,
$$

has only the trivial solution;

ii) The B.V.P. (5.1.20) has only the trivial solution.

iii) The B.V.P. (5.1.4) with boundary conditions

$$
B_{j}\left(\eta, i^{-1} d / d t\right) \phi(\eta, t)_{\mid t=0}=0, \quad j=0, \ldots, q-1,
$$




$$
S_{j}\left(\eta, i^{-1} d / d t\right) \phi(\eta, t)_{\mid t=0}=0, \quad j=0, \ldots, q-1,
$$

has only the trivial solution.

The proof follows by induction.

5.2. A Priori Estimates in the Half-spaces. To proceed further with the scheme of frozen coefficients developed in detail in [LiMa, Ch. 2] we have to consider

1) a priori estimates of one-dimensional B.V.P.,

2) a priori estimates for operators with constant coefficients in the union of the two half-spaces, and

3) a priori estimates for operators with variable coefficients in the union of the two half-spaces.

This may be done by using the results of Section 5.1, Proposition 5.1.1, Lemma 5.1.2, and applying the techniques of the above-mentioned book.

Here we provide the formulation of the final a priori estimate, in the third case only. This is enough to understand the mechanism of the a priori estimates obtained in the present paper.

Next we will consider operators $A$ and $B_{j}, j=0, \ldots, m-1$, such that their principal homogeneous parts are given by (5.1.1)-(5.1.3), i.e. $A_{0}(0, D)=\Delta^{2 q}$, $B_{j, 0}(0, D)=\Delta^{j}, j=0, \ldots, m-1, S_{j, 0}(0, D)=D_{t} B_{j, 0}(0, D), j=0, \ldots, 2 q-1$. by

Let $w=(u, v) \in H^{2 m}\left(\mathbf{R}_{+}^{n}\right) \times H^{2 m}\left(\mathbf{R}_{-}^{n}\right)$. Let us denote by $P$ the operator given

$$
\begin{aligned}
P: w=(u, v) \rightarrow P w=\{ & A(x ; D) u, A(x ; D) v ; \\
& B_{j}(x ; D) u_{\mid t=0}-B_{j}(x ; D) v_{\mid t=0}, j=0, \ldots, m-1 ; \\
& S_{j}(x ; D) u_{\mid t=0}-S_{j}(x ; D) v_{\mid t=0}, j=0, \ldots, m-2 ; \\
& \left.B_{0}(x ; D) u_{\mid t=0}\right\} .
\end{aligned}
$$

Let us introduce the spaces

$$
\begin{gathered}
U_{1, r}=\prod_{j=0}^{m-1} H^{2 m+r-m_{j}-1 / 2}\left(\mathbf{R}^{n-1}\right) \times \prod_{j=0}^{m-2} H^{2 m+r-m_{j}-3 / 2}\left(\mathbf{R}^{n-1}\right) \\
\times H^{2 m+r-1 / 2}\left(\mathbf{R}^{n-1}\right),
\end{gathered}
$$

and

$$
\begin{gathered}
U_{2, r}=\prod_{j=0}^{m-1} H^{-2 m+r+m_{j}+1 / 2}\left(\mathbf{R}^{n-1}\right) \times \prod_{j=0}^{m-2} H^{-2 m+r+m_{j}+3 / 2}\left(\mathbf{R}^{n-1}\right) \\
\times H^{-2 m+r+1 / 2}\left(\mathbf{R}^{n-1}\right),
\end{gathered}
$$

where we have put $m_{j}=2 j=\operatorname{ord}\left(B_{j}\right)$.

Thanks to the theorem about traces [LiMa, Chapter 1.8], the operator $P$ is linear and continuous from

$$
H^{2 m}\left(\mathbf{R}_{+}^{n}\right) \times H^{2 m}\left(\mathbf{R}_{-}^{n}\right) \text { into } L^{2}\left(\mathbf{R}_{+}^{n}\right) \times L^{2}\left(\mathbf{R}_{-}^{n}\right) \times U_{1,0} .
$$

Let us denote by $P^{*}$ the operator adjoint to $P$ which maps $L^{2}\left(\mathbf{R}_{+}^{n}\right) \times L^{2}\left(\mathbf{R}_{-}^{n}\right) \times U_{2,0}$ into $\left[H^{2 m}\left(\mathbf{R}_{+}^{n}\right) \times H^{2 m}\left(\mathbf{R}_{-}^{n}\right)\right]^{\prime}$. 
Theorem 5.2.1. Assume that the operators with constant coefficients $A_{0}(0, D)$, $B_{j, 0}(0, D), j=0, \ldots, m-1, S_{j, 0}(0, D), j=0, \ldots, m-1$, are given by $(5.1 .1)-$ (5.1.3).

Then for every given integer $r \geq 0$ there exists a positive $\rho_{0}$ such that for $\rho<\rho_{0}$ the following statements hold:

i) If $w=(u, v) \in H^{2 m}\left(\mathbf{R}_{+}^{n}\right) \times H^{2 m}\left(\mathbf{R}_{-}^{n}\right)$ is a couple of functions each of which is equal to zero outside the ball $B(0 ; \rho)$ and $P w \in S_{1}=H^{r}\left(\mathbf{R}_{+}^{n}\right) \times H^{r}\left(\mathbf{R}_{-}^{n}\right) \times U_{1, r}$, then $w \in S_{2}=H^{2 m+r}\left(\mathbf{R}_{+}^{n}\right) \times H^{2 m+r}\left(\mathbf{R}_{-}^{n}\right)$ and the following inequality holds:

$$
\|w\|_{S_{2}} \leq C_{r, \rho}\left\{\|P w\|_{S_{1}}+\|w\|_{H^{2 m+r-1}\left(\mathbf{R}_{+}^{n}\right) \times H^{2 m+r-1}\left(\mathbf{R}_{-}^{n}\right)}\right\},
$$

where $C_{r, \rho}$ depends on $r$ and $\rho$.

ii) If $F=\left\{f_{1}, f_{2} ; g_{o}^{1}, \ldots, g_{m-1}^{1} ; g_{o}^{2}, \ldots, g_{m-2}^{2} ; g\right\} \in L^{2}\left(\mathbf{R}_{+}^{n}\right) \times L^{2}\left(\mathbf{R}_{-}^{n}\right) \times U_{2,0}$ is equal to zero outside the ball $B(0 ; \rho)$, i.e. $f_{1}, f_{2}=0$ outside $B(0 ; \rho) ; g_{j}^{1}(y)=0$ for $|y|>\rho, j=0, \ldots, m-1, g_{j}^{2}(y)=0$ for $|y|>\rho, j=0, \ldots, m-2$, and $P^{*} F \in$ $T_{1, r}=H^{-2 m+r}\left(\mathbf{R}^{n}\right) \times H^{-2 m+r}\left(\mathbf{R}^{n}\right)$, then $F \in T_{2, r}=H^{r}\left(\mathbf{R}_{+}^{n}\right) \times H^{r}\left(\mathbf{R}_{-}^{n}\right) \times U_{2, r}$.

For integers $r>0$ we have the inequality

$$
\|F\|_{T_{2, r}} \leq C_{r, \rho}\left\|P^{*} F\right\|_{T_{1, r}}+\|F\|_{H^{r-1}\left(\mathbf{R}_{+}^{n}\right) \times H^{r-1}\left(\mathbf{R}_{-}^{n}\right) \times U_{2, r}},
$$

and for $r=0$, the inequality

$$
\begin{aligned}
\|F\|_{T_{2,0}} \leq C_{0, \rho}\{ & \left.\left\|P^{*} F\right\|_{T_{1,0}}+\left\|\left(\tilde{f}_{1}, \tilde{f}_{2}\right)\right\|_{H^{-1}\left(\mathbf{R}^{n}\right) \times H^{-1}\left(\mathbf{R}^{n}\right)}\right\} \\
& +\sum_{j=0}^{m-1}\left\|g_{j}^{1}\right\|_{H^{-2 m+m_{j}-1 / 2}\left(\mathbf{R}^{n-1}\right)}+\sum_{j=0}^{m-2}\left\|g_{j}^{2}\right\|_{H^{-2 m+m_{j}+1 / 2}\left(\mathbf{R}^{n-1}\right)} \\
& \left.+\|g\|_{H^{-2 m-1 / 2}\left(\mathbf{R}^{n-1}\right)}\right\} .
\end{aligned}
$$

Here $\tilde{f}_{1}$ (resp. $\tilde{f}_{2}$ ) is the continuation of $f_{1}$ (resp. $f_{2}$ ) as zero for $t<0$ (resp. $t>0)$.

The proof uses the same techniques as the proof of [LiMa, Ch. 2, Theorem 4.3] and is based on Proposition 5.1.1 and Lemma 5.1.2.

\section{A Priori Estimates and Existence in a Bounded Domain}

Here we provide a priori estimates for a bounded domain with interior boundaries in order to use them for proving a Fredholm alternative.

\subsection{A Priori Estimates in a Bounded Domain with Interior Boundaries.}

Let us go back to the interior B.V.P. (3.1)-(3.4).

We put $m=2 q$ and assume all notations of Sections 2,3 .

We will consider the more general boundary value problem

$$
A u(x)=f(x), \quad x \in D \backslash S T ;
$$

$$
\begin{gathered}
B_{p} u_{N}(x)=h_{p}(x), \quad x \in T_{N}=\partial D, \quad \text { for } p=1, \ldots, q-1 ; \\
S_{p} u_{N}(x)=h_{q+p}(x), \quad x \in T_{N}=\partial D, \quad \text { for } p=0, \ldots, q-1 ;
\end{gathered}
$$

for $p=0, \ldots, m-1, j=1, \ldots, N-1$;

$$
S_{p} u_{j}(x)=S_{p} u_{j+1}(x)+g^{2}(x), \quad x \in T_{j},
$$


for $p=0, \ldots, m-2, j=1, \ldots, N-1$;

$$
B_{0} u_{j}(x)=g(x), \quad x \in T_{j},
$$

for $j=1, \ldots, N$;

Here $A=\Delta^{2 q}$ in the whole of the domain $D \backslash S T$. The operators $B_{p}$ are given by $B_{p} u_{j}=\Delta^{p} u_{j}, x \in T_{j} \cup T_{j-1}$, for $p=0, \ldots, m-1$, for every domain $D_{j}, j=$ $1, \ldots, N$, and function $u_{j}$ defined there. The operators $S_{p}$ are given by the equality

$$
\begin{gathered}
S_{p} u_{j}=\left(\partial / \partial n_{j}\right) B_{p} u_{j}(x), \quad x \in T_{j}, \\
S_{p} u_{j}=\left(\partial / \partial n_{j-1}\right) B_{p} u_{j}(x), \quad x \in T_{j-1},
\end{gathered}
$$

for $p=0, \ldots, m-1$, and for every domain $D_{j}, j=1, \ldots, N$, and function $u_{j}$ defined there.

We assume that the domain $D$ is bounded with boundary $\partial D=T_{N}$ which is $C^{\infty}$, and locally $D$ is on one side of $\partial D$. Each surface $T_{j}, j=1, \ldots, N-1$, is an infinitely smooth manifold of dimension $n-1$, such that the domain surrounded by $T_{j}$ is on one side of $T_{j}$.

Let us put $S T_{1}=\bigcup_{j=1}^{N-1} T_{j}, \quad m_{j}=\operatorname{ord}\left(B_{j}\right)=2 j$ for $j=0, \ldots q-1$, and $m_{j}=\operatorname{ord}\left(S_{j-q}\right)=2(j-q)+1$ for $j=q, \ldots, 2 q$.

To simplify the writing let us put

$$
\begin{aligned}
U_{1, r}= & \prod_{j=1}^{m-1} H^{2 m+r-m_{j}-1 / 2}(\partial D) \times \prod_{j=0}^{m-1} H^{2 m+r-m_{j}-1 / 2}\left(S T_{1}\right) \\
& \times \prod_{j=0}^{m-2} H^{2 m+r-m_{j}-3 / 2}\left(S T_{1}\right) \times H^{2 m+r-1 / 2}(S T), \\
U_{2, r}= & \prod_{j=1}^{m-1} H^{-2 m+r+m_{j}+1 / 2}(\partial D) \times \prod_{j=0}^{m-1} H^{-2 m+r+m_{j}+1 / 2}\left(S T_{1}\right) \\
& \times \prod_{j=0}^{m-2} H^{-2 m+r+m_{j}+3 / 2}\left(S T_{1}\right) \times H^{-2 m+r+1 / 2}(S T) .
\end{aligned}
$$

We consider the data vector of problem (6.1.1)-(6.1.5) given by

$$
F=\left\{f, h ; g_{0}^{1}, \ldots, g_{m-1}^{1} ; g_{0}^{2}, \ldots, g_{m-2}^{2} ; g\right\} \in L^{2}(D \backslash S T) \times U_{2,0} .
$$

The corresponding operator given by

$$
P: u \rightarrow F
$$

is linear and continuous from $H^{2 m}(D \backslash S T)$ into $L^{2}(D \backslash S T) \times U_{1,0}$.

Let us denote by $P^{*}$ the operator adjoint to $P$ mapping $L^{2}(D \backslash S T) \times U_{2,0}$ into $\left[H^{2 m}(D \backslash S T)\right]^{\prime}$, which is defined by the equality

$$
\left\langle u, \overline{P^{*} F}\right\rangle=\langle P u, \bar{F}\rangle,
$$

true for every $F \in L^{2}(D \backslash S T) \times U_{2,0}$ and every $u \in H^{2 m}(D \backslash S T)$; here the brackets

$\langle$,$\rangle denote the pairing between the corresponding dual spaces.$

We now give the main a priori estimate: 
Theorem 6.1.1. Under the above assumptions, for every given integer $r \geq 0$ the following statements are true:

$\left.1^{0}\right)$ If $u \in H^{2 m}(D \backslash S T)$ and $P u \in H^{r}(D \backslash S T) \times U_{1, r}$, then $u \in H^{2 m+r}(D \backslash S T)$ and

$$
\|u\|_{H^{2 m+r}(D \backslash S T)} \leq C_{r}\left\{\|P u\|_{H^{r}(D \backslash S T) \times U_{1, r}}+\|u\|_{H^{2 m+r-1}(D \backslash S T)}\right\},
$$

where $C_{r}$ depends on $r$.

$\left.2^{0}\right)$ If $F=\left\{f ; h ; g_{0}^{1}, \ldots, g_{m-1}^{1} ; g_{0}^{2}, \ldots, g_{m-2}^{2} ; g\right\} \in L^{2}(D \backslash S T) \times U_{2,0}$ and $P^{*} F \in$ $\left[H^{2 m-r}(D \backslash S T)\right]^{\prime}$, then $F \in H^{r}(D \backslash S T) \times U_{2, r}$.

For integers $r>0$

$$
\|F\|_{H^{r}(D \backslash S T) \times U_{2, r}} \leq C_{r}\left\{\left\|P^{*} F\right\|_{\left[H^{2 m-r}(D \backslash S T)\right]^{\prime}}+\|F\|_{H^{r-1}(D \backslash S T) \times U_{2, r}}\right\},
$$

and for $r=0$

$$
\begin{aligned}
\|F\|_{L^{2}(D \backslash S T) \times U_{2,0}} \leq C_{0}\{ & \left\|P^{*} F\right\|_{\left[H^{2 m}(D \backslash S T)\right]^{\prime}}+\|\tilde{f}\|_{\left[H^{-1}\left(\mathbf{R}^{n}\right)\right]^{N}} \\
& +\sum_{p=0}^{m-1}\left\|g_{p}^{1}\right\|_{H^{-2 m+m_{p}-1 / 2}(S T)} \\
& \left.+\sum_{p=0}^{m-2}\left\|g_{p}^{2}\right\|_{H^{-2 m+m_{p}+1 / 2}(S T)}+\|g\|_{H^{-2 m-1 / 2(S T)}}\right\},
\end{aligned}
$$

where by $\tilde{f}$ we denote the set of $N$ functions $\tilde{f}_{j}, j=1, \ldots, N$, such that $\tilde{f}_{j}$ is a continuation like zero of the function $f_{j}=f_{\mid D_{j}}$ outside the domain $D_{j}$.

The proof uses a partition of unity, and

1) the a priori estimate of [LiMa, Theorem 2.5.1, Ch.2] at interior points of $D \backslash S T$;

2) the a priori estimate of [LiMa, Theorem 3.1, Ch.2] at boundary points of $\partial \mathrm{D}$. This is possible since the system of boundary operators $S_{j}, B_{j}, j=0, \ldots, q-1$, satisfy the Agmon-Douglis-Nirenberg condition with respect to the operator $A=$ $\Delta^{2 q}$, see Lemma 5.1.2, iii) above;

3 ) the a priori estimate near the interior boundary points of $S T_{1}$ provided by Theorem 5.2.1.

6.2. Existence of a Solution in the Spaces $H^{s}(D \backslash S T)$ for Integers $s \geq$ $2 m$. Now we may study the boundary value problem (6.1.1)-(6.1.5) in the spaces $H^{2 m+r}(D \backslash S T)$ for integers $r \geq 0$. We have to prove that the operator given by (6.1.6) is a Fredholm operator from $H^{2 m+r}(D \backslash S T)$ into $H^{r}(D \backslash S T) \times U_{1, r}$, assuming the notations of the previous paragraph.

Recall that the index $\chi(P)$ of $P$ is given by the formula

$$
\chi(P)=\operatorname{dim}(\operatorname{Ker}(P))-\operatorname{codim}(\operatorname{Im}(P)) .
$$

Using the standard argumentation based on Peetre's lemma, as in [LiMa, Theorem 5.2, Ch. 2] we have the following:

Theorem 6.2.1. Let the assumptions of Section 6.1 hold. Then the operator $P$ defined by (6.1.6), considered from $H^{2 m+r}(D \backslash S T)$ into $H^{r}(D \backslash S T) \times U_{1, r}$, for $r=0,1,2, \ldots$, has an index $\chi(P)$ which does not depend on $r$. The kernel of $P$ is the space

$$
\Lambda=\left\{u: u_{\mid D_{j}}=u_{j} \in \mathcal{D}\left(\bar{D}_{j}\right), P u=0\right\}
$$


and its image is the set of elements $F=\left\{f ; h ; g_{0}^{1}, \ldots, g_{m-1}^{1} ; g_{0}^{2}, \ldots, g_{m-2}^{2} ; g\right\}$ of the space $H^{r}(D \backslash S T) \times U_{1, r}$ such that

$$
\langle F, \Phi\rangle=0
$$

for every element $\Phi=\left\{v ; \chi ; \gamma_{0}^{1}, \ldots, \gamma_{m-1}^{1} ; \gamma_{0}^{2}, \ldots, \gamma_{m-2}^{2} ; \gamma\right\}$ of the space

$$
\Lambda_{1}=\left\{\Phi \in L: \text { such that } P^{*} \Phi=0\right\} \text {, }
$$

where we have put

$$
\begin{aligned}
L= & \prod_{j=1}^{N} \mathcal{D}\left(\overline{D_{j}}\right) \times[\mathcal{D}(\partial D)]^{m-1} \\
& \times\left[\mathcal{D}\left(S T_{1}\right)\right]^{m} \times\left[\mathcal{D}\left(S T_{1}\right)\right]^{m-1} \times[\mathcal{D}(S T)]^{m},
\end{aligned}
$$

and $P^{*}$ is the operator adjoint to $P$, and is defined through the duality relation (6.1.7) (where $r=0$ ).

The following proposition explicitly describes the space $\Lambda_{1}$, and the consistency conditions (6.2.2) providing solubility of problem (6.1.1)-(6.1.5).

Proposition 6.2.2. The space $\Lambda_{1}$ defined by (6.2.3) coincides with the set run by the element

$$
\begin{gathered}
\Phi=\left\{v ;-S_{m-1-p} v_{N \mid T_{N}}, p=1, \ldots, q-1 ;\right. \\
-B_{m-1-p} v_{N \mid T_{N}}, p=0, \ldots, q-1 ; \\
-S_{m-1} v_{j+1 \mid T_{j}}, j=1, \ldots, N-1 ; \\
-S_{m-1-p} v_{j \mid T_{j}}, p=1, \ldots, m-1, j=1, \ldots, N-1 ; \\
B_{m-1-p} v_{j \mid T_{j}}, p=0, \ldots, m-2, j=1, \ldots, N-1 ; \\
\left.\left(-S_{m-1} v_{j}+S_{m-1} v_{j+1}\right)_{\mid T_{j}}, j=1, \ldots, N-1 ;-S_{m-1} v_{N \mid T_{N}}\right\}
\end{gathered}
$$

where $v$ is such that $v_{\mid D_{j}} \in \mathcal{D}\left(\overline{D_{j}}\right)$ and

$$
\begin{gathered}
A v(x)=A^{*} v(x)=0, \quad x \in D \backslash S T ; \\
B_{p} v_{N}(x)=0, \quad x \in T_{N}=\partial D, p=1, \ldots, q-1 ; \\
S_{p} v_{N}(x)=0, \quad x \in T_{N}=\partial D, p=0, \ldots, q-1 ; \\
B_{p} v_{j}(x)-B_{p} v_{j+1}(x)=0, \quad x \in T_{j}, p=0, \ldots, m-1, j=1, \ldots, N-1 ; \\
S_{p} v_{j}(x)-S_{p} v_{j+1}(x)=0, \quad x \in T_{j}, p=0, \ldots, m-2, j=1, \ldots, N-1 ; \\
B_{0} u_{j}(x)=0, \quad x \in T_{j}, j=1, \ldots, N .
\end{gathered}
$$


The proof is based on the following Green's formula obtained from [Aron, p. 9] or [LiMa, Ch.2, Sec. 2]:

$$
\int_{D_{j}}\left\{u \Delta^{2 q} v-\Delta^{2 q} u v\right\} d x+\sum_{p=0}^{m-1} \int_{\partial D_{j}}\left(B_{p} u S_{2 q-1-p} v-S_{p} u B_{2 q-1-p} v\right)=0 .
$$

It also relies upon the fact that the system of boundary operators $\left\{B_{0}, \ldots, B_{m-1}\right.$, $\left.S_{0}, \ldots, S_{m-1}\right\}$ forms a Dirichlet system on every boundary $T_{j}, j=1, \ldots, N$, i.e. the set of their orders exhausts the set of numbers $\{0,1, \ldots, 2 m-1\}$. The last permits the application of Lemma 2.2.2 from [LiMa, Chapter 2], which gives that the vector

$$
\left\{B_{p} u_{j \mid T_{j-1}}, B_{p} u_{j \mid T_{j}}, S_{p} u_{j \mid T_{j-1}}, S_{p} u_{j \mid T_{j}}\right\}_{p=0}^{m-1}
$$

runs through the space $\left[\mathcal{D}\left(T_{j} \cup T_{j-1}\right)\right]^{2 m}$.

Here we give more information about the consistency conditions (6.2.2)-(6.2.3).

Corollary 6.2.3. The boundary value problem (6.1.1)-(6.1.5) is self-adjoint, and for the space defined by (6.2.3) we have $\Lambda_{1}=\{0\}$.

Proof. Proposition 6.2.2 in fact provides a description of the B.V.P. adjoint to (6.1.1)-(6.1.5), which evidently coincides with it. On the other hand, according to Theorem 4.1 we have unique solubility to B.V.P. (6.1.1)-(6.1.5). Consequently, the adjoint problem is uniquely solvable as well, and representation (6.2.3) implies that $\Lambda_{1}=\{0\}$.

Q.E.D

Here is the existence result:

Theorem 6.2.4. Let the assumptions of Section 6.1 hold. Then for the operator $P$, given by (6.1.6), considered as an operator from $H^{2 m+r}(D \backslash S T)$ into $H^{r}(D \backslash S T) \times U_{1, r}$, for $r=0,1,2, \ldots$, we have $\operatorname{Ker}(P)=\{0\}$ and $\operatorname{codim}(\operatorname{Im}(P))$ $=0$, i.e. $P$ is a Fredholm operator and has an index $\chi(P)=0$. Hence, B.V.P. (6.1.1)-(6.1.5) is solvable for all right sides from the indicated spaces.

The proof follows immediately from Corollary 6.2.3 and Theorem 6.2.1.

6.3. Existence of Polysplines. Let us go back to our original problem, the existence of interpolation polysplines, given by (3.1)-(3.4), and satisfying (5.1). As an immediate corollary of Theorem 6.2.4 we obtain

Theorem 6.3.1. Let us assume that in the domain $D \subseteq \mathbf{R}^{n}$ we have a family of monotonely imbedded closed surfaces $T_{j}, j=1, \ldots, N$, with $T_{N}=\partial D$, which are defined in Section 2. Assume that the surfaces $T_{j}$ have the smoothness properties provided in Section 6.1. Then for every integer $r \geq 0$ and data $g_{j} \in H^{4 q+r-1 / 2}\left(T_{j}\right), j=$ $1, \ldots, N$, there exists a unique interpolation polyspline $u(x)$, a solution to problem (3.1)-(3.4), (5.1), such that its pieces satisfy $u_{j} \in H^{4 q+r}\left(\overline{D_{j}}\right), j=1, \ldots, N$, and thus $u \in H L_{2 q-1}(D)$.

\section{Examples And Applications}

The main purpose of the examples below is to see that the computation of the interpolation polysplines in some particular cases of a data set $S T$ possessing symmetry is reduced to finding one-dimensional interpolation $L$-splines. That shows on one hand ways to compute the polysplines numerically; on the other hand it proves that, in a certain sense, the polysplines are a genuine generalization of the 
one-dimensional concept of spline. We restrict ourselves to the formal side of the computations, without discussing in detail the convergence of the Fourier series or integrals that appear.

1. Data set on concentric circles and biharmonic polysplines.

Let us set

$$
T_{j}=\left\{\left(x_{1}, x_{2}\right)=(r, \theta) \in R^{2}:|x|=r=r_{j}\right\}, \quad j=1, \ldots, N,
$$

for the numbers $0=r_{0}<r_{1}<\ldots<r_{N}$, and the polar coordinates $(r, \theta)$.

Condition (3.1) states that the biharmonic polyspline satisfies

$$
\Delta^{2} u_{j}(x)=0, \quad r_{j-1}<|x|<r_{j}, \quad j=1, \ldots, N
$$

for $u_{1}$ the above holds for $x=0$ as well.

From (3.3), with $p=0,1$, we obtain

$$
\begin{gathered}
u_{j}\left(r_{j}, \theta\right)=u_{j+1}\left(r_{j}, \theta\right)=g_{j}(\theta), \quad \theta \in[0,2 \pi], \quad j=1, \ldots, N-1 ; \\
\Delta u_{j}\left(r_{j}, \theta\right)=\Delta u_{j+1}\left(r_{j}, \theta\right), \quad \theta \in[0,2 \pi], \quad j=1, \ldots, N-1 .
\end{gathered}
$$

From (3.4), with $p=0$, we obtain

$$
\frac{\partial u_{j}}{\partial r}\left(r_{j}, \theta\right)=\frac{\partial u_{j+1}}{\partial r}\left(r_{j}, \theta\right), \quad \theta \in[0,2 \pi], \quad j=1, \ldots, N-1 .
$$

Since the Laplace operator has the form

$$
\Delta f=\frac{\partial^{2} f}{\partial r^{2}}+\frac{1}{r} \frac{\partial f}{\partial r}+\frac{1}{r^{2}} \frac{\partial^{2} f}{\partial \theta^{2}}
$$

and if we assume that $g_{j} \in C^{2}\left(T_{j}\right)$, it follows from the second equality in (7.2) that

$$
\frac{\partial^{2} u_{j}}{\partial r^{2}}\left(r_{j}, \theta\right)=\frac{\partial^{2} u_{j+1}}{\partial r^{2}}\left(r_{j}, \theta\right), \quad \theta \in[0,2 \pi], \quad j=1, \ldots, N-1 .
$$

Further we make a Fourier transfrom in $\theta$ and put, for every integer $\tau$,

$$
v_{j}(r, \tau)=\widehat{u_{j}}(r, \tau)=\int_{0}^{2 \pi} u_{j}(r, \theta) e^{-i \theta \tau} d \theta .
$$

Let $\widehat{g_{j}}(\tau)$ denote the Fourier transform of the data function $g_{j}(\theta)$. For every integer $\tau$ the above equalities become

$$
\begin{array}{rlrl}
\left(\frac{\partial^{2}}{\partial r^{2}}+\frac{1}{r} \frac{\partial}{\partial r}-\frac{\tau^{2}}{r^{2}}\right)^{2} v_{j}(r, \tau) & =0, \quad r_{j-1}<r<r_{j}, & & j=1, \ldots, N \\
v_{j}\left(r_{j}, \tau\right) & =v_{j+1}\left(r_{j}, \tau\right)=\widehat{g_{j}}(\tau), & & j=1, \ldots, N-1 ; \\
\frac{\partial v_{j}}{\partial r}\left(r_{j}, \tau\right) & =\frac{\partial v_{j+1}}{\partial r}\left(r_{j}, \tau\right), & j=1, \ldots, N-1 ; \\
\frac{\partial^{2} v_{j}}{\partial r^{2}}\left(r_{j}, \tau\right) & =\frac{\partial^{2} v_{j+1}}{\partial r^{2}}\left(r_{j}, \tau\right), & & j=1, \ldots, N-1 .
\end{array}
$$

It follows that in the interval $\left(0, r_{N}\right)$ we have an $L$-spline of order 4 defined by the operator

$$
L=\left(\frac{\partial^{2}}{\partial r^{2}}+\frac{1}{r} \frac{\partial}{\partial r}-\frac{\tau^{2}}{r^{2}}\right)^{2} .
$$

After the change $v=2 \log (r)$ we get a well known one-dimensional problem [Schum, Chapter 10, and references there]. After finding its solution for every integer $\tau$, we obtain the polyspline $u_{j}(r, \theta)$ by expanding it in a Fourier series. 
For simplicity we considered only the plane $\mathbf{R}^{2}$, and biharmonic polysplines. The computations are essentially the same if we expand the polyspline components $u_{j}(x)$ in spherical harmonics for arbitrary space dimension $n$ and order $2 q$.

To make things even more comprehensive we shall consider the case of spherically symmetric biharmonic polysplines in $\mathbf{R}^{2}$, i.e. when the data $g_{j}$ are radially symmetric: $g_{j}(\theta)=$ Const $=g_{j}, \theta \in[0,2 \pi], j=1, \ldots, N$. Then the functions $u_{j}(r, \theta)$ depend only on $r$.

In [Vek, p. 181, formulas (35.29-32)] one may find the representation formulas for functions biharmonic in a layer. Using them, we find that $u_{j}$ may be written as

$$
u_{j}(x)=A_{j}+B_{j} r^{2}+\left(C_{j}+D_{j} r^{2}\right) \log (r), \quad r=|x|,
$$

for $j=1,2, \ldots, N$, where $A_{j}, B_{j}, C_{j}, D_{j}$ are constants, and $C_{1}=D_{1}=0$.

Besides the above conditions (7.1)-(7.3), we have also a boundary condition in (3.2) which reduces to

$$
\frac{\partial u_{N}\left(r_{N}\right)}{\partial r}=0 .
$$

The interpolation condition (5.1) becomes

$$
u\left(r_{j}\right)=g_{j}, \quad j=1, \ldots, N .
$$

Clearly, we have to see that there exist constants $A_{j}, B_{j}, C_{j}, D_{j}$ such that the above interpolation and boundary conditions (7.2)-(7.5) are fulfilled.

In order to demonstrate how the main idea of the present paper works in that simple situation, we shall give a direct proof of the existence Theorem 6.3.1.

For that purpose, introduce the operator

$$
S_{r} f(r)=r^{1 / 2} \Delta_{r} f=r^{-1 / 2}(\partial / \partial r)(r \partial f / \partial r) .
$$

Lemma 7.1. Let $u$ be the biharmonic polyspline defined above. Then for every function $g \in H^{2}\left(0, r_{N}\right)$ the following identity holds:

$$
\begin{gathered}
\int_{0}^{r_{N}} S_{r} u(r) S_{r} g(r) d r=\int_{0}^{r_{N}} r\left[\Delta_{r}\right]^{2} u(r) g(r) d r \\
+\sum_{k=1}^{N} \lambda_{k}\left(\partial^{3} u\left(r_{k}+0\right) / \partial r^{3}-\partial^{3} u\left(r_{k}-0\right) / \partial r^{3}\right) g\left(r_{k}\right) \\
+r_{N}(\partial / \partial r) \Delta_{r} u_{N}\left(r_{N}\right) g\left(r_{N}\right)+(\partial / \partial r) r(\partial / \partial r) u_{N}\left(r_{N}\right)(\partial / \partial r) g\left(r_{N}\right),
\end{gathered}
$$

where the $\lambda_{k}$ are some appropriate constants.

The proof easily follows through integration by parts.

Let us prove uniqueness for the interpolation problem (7.5).

If $g_{j}=0, j=1, \ldots, N$, then we put $g=u$ in the above identity, and obtain

$$
\int_{0}^{r_{N}}\left(S_{r} u(r)\right)^{2} d r=0
$$

This implies $S_{r} u\left(r_{j}\right)=0, j=1, \ldots, N$, or $\Delta_{r} u\left(r_{j}\right)=0$. Since $u_{j}\left(r_{j}\right)=u_{j}\left(r_{j-1}\right)=$ 0 for $j=2, \ldots, N$, it follows that $u_{j} \equiv 0$. 
Thus we have proved that for every right side $\left\{g_{j}\right\}_{j=1}^{N}$, there exists a unique $u$ satisfying (7.1)-(7.5). Since the system (7.1)-(7.5) is linear with respect to the constants $A_{j}, B_{j}, C_{j}, D_{j}$, the uniqueness implies existence of $u$.

2. Data set on parallel hyperplanes and biharmonic polysplines.

Let $S T$ be a set of parallel hyperplanes in $\mathbf{R}^{n}$, i.e.

$$
T_{j}=\left\{(x, t) \in \mathbf{R}^{n}: x \in \mathbf{R}^{n-1}, t=t_{j}\right\}, \quad j=1, \ldots, N,
$$

where the numbers $t_{1}<\ldots<t_{N}$. Here $u_{j}(x, t)$ is defined in the layer between the hyperplanes $T_{j-1}$ and $T_{j}$. As in the first example, conditions (3.2)-(3.4) simplify. Let us make a Fourier transform in the variable $x$ by putting, for every $\xi \in \mathbf{R}^{n-1}$,

$$
v_{j}(\xi, t)=\widehat{u_{j}}(\xi, t)=\int_{R^{n-1}} u_{j}(x, t) e^{-i x \xi} d x,
$$

and let $\widehat{g_{j}}(\xi)$ denote the Fourier transfrom of the data $g_{j}(x)$.

For every $\xi \in \mathbf{R}^{n-1}$ the main conditions in (3.1)-(3.4) (without the boundary ones) become

$$
\begin{aligned}
\left(\frac{\partial^{2}}{\partial t^{2}}-|\xi|^{2}\right)^{2} v_{j}(\xi, t) & =0, \quad t_{j-1}<t<t_{j}, & & j=2, \ldots, N ; \\
v_{j}\left(\xi, t_{j}\right) & =v_{j+1}\left(\xi, t_{j}\right)=\widehat{g}_{j}(\xi), & & j=2, \ldots, N-1 ; \\
\frac{\partial v_{j}}{\partial t}\left(\xi, t_{j}\right) & =\frac{\partial v_{j+1}}{\partial t}\left(\xi, t_{j}\right), & & j=2, \ldots, N-1 ; \\
\frac{\partial^{2} v_{j}}{\partial t^{2}}\left(r_{j}, t_{j}\right) & =\frac{\partial^{2} v_{j+1}}{\partial t^{2}}\left(\xi, t_{j}\right), & & j=2, \ldots, N-1 .
\end{aligned}
$$

Consequently, for every $\xi \in \mathbf{R}^{n-1}$, the function $v(\xi, t)$ is a one-dimensional interpolation $L$-spline defined by the operator $L=\left(\frac{\partial^{2}}{\partial t^{2}}-|\xi|^{2}\right)^{2}$, which is computable by the reference quoted in the previous example. The original polyspline components $u_{j}(x, t)$ are obtained by applying the inverse Fourier transform to $v_{j}(\xi, t)$. The case of arbitrary order $2 q$ polysplines was studied in [Ko4].

Let us remark that the existence result for polysplines proved in Theorem 6.3.1 does not apply directly to the case of unbounded domains, which is the case of the above example. Here we may profit from the results in Section 5. Namely, if we assume as in Theorem 5.2.1 that the data functions $g_{j}, j=1, \ldots, N$, have compact supports and belong to $H^{3 \frac{1}{2}}\left(T_{j}\right)$, the convergence of the inverse Fourier transform above will follow automatically, and also the smoothness of the polysplines obtained will be, near the interior boundary $S T$, that of Theorem 6.3.1.

3. Data set on rays and biharmonic polysplines.

Let $S T$ be a set of rays in $\mathbf{R}^{n}$ coming out of the origin, i.e.

$$
T_{j}=\left\{\left(x_{1}, x_{2}\right) \in \mathbf{R}^{2}: \theta=\theta_{j}\right\}, \quad j=1, \ldots, N,
$$

where $(r, \theta)$ are the polar coordinates of $\left(x_{1}, x_{2}\right)$, and $0=\theta_{0}<\theta_{1}<\ldots<\theta_{N-1}<$ $\theta_{N}=2 \pi$. Let $u_{j}$ be defined for $\theta_{j-1} \leq \theta \leq \theta_{j}, j=1, \ldots, N$. For simplicity we put $u_{N+1}=u_{1}$.

Now we make the following transform: we put $t=-\ln r$ and make the Fourier transform $t \rightarrow \lambda$. The whole transform is the so-called Mellin transform. Let us denote by $v_{j}(\lambda, \theta)$ the image of $u_{j}(r, \theta)$. 
Then as in the previous cases, for every $\lambda \in \mathbf{R}^{1}$, conditions (3.1)-(3.4) become

$$
\begin{gathered}
\left(\frac{\partial^{2}}{\partial \theta^{2}}-\lambda^{2}\right)\left(\frac{\partial^{2}}{\partial \theta^{2}}-(\lambda-2 i)^{2}\right) v_{j}(\lambda, \theta)=0, \quad \theta_{j-1} \leq \theta \leq \theta_{j} \\
v_{j}\left(\lambda, \theta_{j}\right)=v_{j+1}\left(\lambda, \theta_{j}\right)=\widehat{g_{j}}(\lambda) \\
\frac{\partial v_{j}}{\partial \theta}\left(\lambda, \theta_{j}\right)=\frac{\partial v_{j+1}}{\partial \theta}\left(\lambda, \theta_{j}\right) \\
\frac{\partial^{2} v_{j}}{\partial \theta^{2}}\left(\lambda, \theta_{j}\right)=\frac{\partial^{2} v_{j+1}}{\partial \theta^{2}}\left(\lambda, \theta_{j}\right)
\end{gathered}
$$

all for $j=1, \ldots, N$.

Consequently, for every $\lambda \in \mathbf{R}^{1}$, the function $v(\lambda, \theta)$ is a periodic interpolation $L$-spline defined by the operator

$$
L=\left(\frac{\partial^{2}}{\partial \theta^{2}}-\lambda^{2}\right)\left(\frac{\partial^{2}}{\partial \theta^{2}}-(\lambda-2 i)^{2}\right),
$$

and may be computed by the previous reference to [Schum].

The original polyspline $u_{j}(r, \theta)$ is obtained by the inverse Mellin transform.

That example may be generalized to the case of $S T$ which is a set of hyperplanes in $\mathbf{R}^{n}$ which intersect at $\mathbf{R}^{n-2}$.

We have to make a remark similar to that after example 2). In order to obtain the smoothness rate of Theorem 6.3.1, example 3 needs some additional conditions on the data related to the singularity of the interior boundary $S T$. Let us mention that the problem was treated in [Ko3].

Finally, we want to point out the limits of application of Theorem 6.3.1 which are imposed by the Sobolev imbedding theorem, by considering a particular case.

4. Let us apply Theorem 6.3.1 to the simplest case, that of biharmonic polysplines in the plane, where $T_{j}, j=1,2$, are two circles as in example 1 . Accordingly, the data are two functions $g_{1}, g_{2}$ defined on $T_{1}, T_{2}$ respectively. Let us remark that the solution may be obtained by following the procedure explained in example 1 . Theorem 6.3.1 states that the condition $g_{j} \in H^{3 \frac{1}{2}}\left(T_{j}\right)$ is necessary and sufficient in order to obtain a solution $u \in H^{4}(D \backslash S T)$ which is smooth up to the interior boundary $S T$, i.e. $u_{j} \in H^{4}\left(\overline{D_{j}}\right)$. Since $T_{j}, j=1,2$, are one-dimensional manifolds, $\partial D_{1}=T_{1}, \partial D_{2}=T_{1} \cup T_{2}$, by the Sobolev embedding theorem (cf. [Adams], p. 97, Theorem 5.4) it follows that $g_{1} \in C^{2}\left(T_{1}\right), g_{2} \in C^{2}\left(T_{1}\right), g_{2} \in C^{2}\left(T_{2}\right)$.

This restriction on the data is present also in the Hölder space approach modelled after $[\mathrm{ADN}]$. In that setting smoothness of the solution up to the boundary also requires sufficient smoothness of the data.

In fact, the result of Theorem 6.3.1 may be easily extended to Sobolev spaces of arbitrary real exponent $s \geq 4 q$ following the scheme of [LiMa, Chapter 2.5.4], and for all other real exponents $s$, including $s \leq 0$, by following the scheme in [LiMa, Chapter 2, Sections 7,8]. Thus we will have a solution for data $g_{j}$ belonging to arbitrary Sobolev space $H^{s-\frac{1}{2}}\left(T_{j}\right)$. In that case the polyspline $u$ itself will belong to a Sobolev space (denoted by $\Xi$ in $[\mathrm{LiMa}]$ ) of exponent $s$. For example, let us take $g_{2} \equiv 0, g_{1}\left(x_{1}, x_{2}\right)=0, x_{1} \leq 0$ and $g_{1}\left(x_{1}, x_{2}\right)=1, x_{1}>0$, i.e. data which are piecewise smooth with simple jumps. We have that $g_{j} \in H^{\frac{1}{2}-\epsilon}\left(T_{j}\right)$ for every $\epsilon>0$; see, e.g., [LiMa, Chapter 2, Theorem 10.2]. By the above remarks the solution will belong to a Sobolev space of exponent $1-\epsilon$ in both domains $D_{1}, D_{2}$ up to the 
boundary! It does not seem to be of much practical use though, due to its low smoothness near the boundary $T_{1} \cup T_{2}$.

From the point of view of practical smoothing techniques, the reasonable alternative, in the case of arbitrary data functions $g_{j}$, is to approximate the last through smoother data functions, namely $\overline{g_{j}} \in H^{3 \frac{1}{2}}\left(T_{j}\right)$, and find the corresponding interpolation biharmonic polyspline for the data $\overline{g_{j}}$. This would at least provide a sufficiently smooth "approximate" interpolation polyspline $u$.

\section{ACKNOWLEDGEMENTS}

The author is very grateful to Professor Borislav Bojanov at Sofia University for inspiring conversations about splines. Thanks are due to Professor Georgi Karadzhov, for fruitful discussions on elliptic boundary value problems. The unprejudiced (from the point of view of splines) reading of the manuscript by Professor Joseph Bennish is acknowledged with thanks. We extend our thanks to the anonymous referee, whose questions and remarks have motivated us to supply the paper with several illustrative examples, making it more understandable for people with concrete thinking.

\section{REFERENCES}

[Adams] R. A. Adams, Sobolev spaces, Academic Press, New York, 1975. MR 56:9247

[ADN] S. Agmon, A. Douglis, L. Nirenberg, Estimates near the boundary for solutions of elliptic partial differential equations satisfying general boundary conditions, I, Comm. Pure Appl. Math., 12 (1959), 623-727; part II, Comm. Pure Appl. Math., 17 (1964), 35-92. MR 23:A2610; MR 28:5252

[ANW] J. Ahlberg, E. Nilson, J. Walsh, The Theory of Splines and Their Applications, Academic Press, N.Y., 1967. (Russian transl. "Mir", Moscow, 1972) MR 39:684

[Aron] N. Aronszajn, T.M. Creese, L.J.Lipkin, Polyharmonic Functions, Clarendon Press, Oxford, 1983. MR 86g:31001

[Bri] J.C. Briggs, Machine contouring using minimum curvature, Geophysics, 39 (1974), 39-48.

$[\mathrm{Du}] \mathrm{J}$. Duchon, Interpolation des fonctions de deux variables suivant le principe de la flexion des plaques minces, R.A.I.R.O. Analyse Numérique, vol. 10, no. R-3 (1976), pp. 5-12. MR 57:10315

[FJ] S. Fisher, J. Jerome, Minimum Norm Extremals in Function Spaces, Lecture Notes in Math., No. 479, Springer- Verlag, Berlin, 1975. MR 56:1159

[GA] P. Gonzalez-Casanova and R. Alvarez, Splines in geophysics, Geophysics, 50, No. 12 (1985), 2831-2848.

[H] L. Hörmander, The Analysis of Linear Partial Differential Operators III. PseudoDifferential Operators, Springer-Verlag, Berlin-Heidelberg-New York-Tokyo, 1985. MR 87d:35002a

[HK] W. Haussmann, O.I. Kounchev, Peano theorem for linear functionals vanishing on polyharmonic functions, In: Approximation Theory VIII, Vol. 1, Charles Chui and L. Schumaker (eds.), World Scientific Publishing Co., 1995, pp. 233-240.

[Ko1] O.I. Kounchev, Definition and basic properties of polysplines, I and II, C. R. Acad. Bulg. Sci., 44 (1991), No. 7, pp. 9-11; No. 8, pp. 13-16. MR 93a:41016; MR 92m:41031

[Ko2] O.I. Kounchev, Multivariate splines constructed through biharmonic functions, In: Abstracts presented at the Meeting of AMS, Tampa, March-1991, 12 (1991), No. 3, 865-41197.

[Ko3] O.I. Kounchev, Theory of polysplines - minimizing the Laplacian of a function squared with prescribed values on interior boundaries with singularities, II, University of Duisburg, preprint SM-DU-212,1993.

[Ko4] O. I. Kounchev, Splines constructed by pieces of polyharmonic functions, In: Wavelets, Images and Surface Fitting, Eds. P.-J. Laurent et al., AK Peters, Mass., 1994, pp. 319-326. 
[Ko5] O.I. Kounchev, Minimizing the integral of the Laplacian of a function suqared with prescribed values on interior boundaries - theory of polysplines, I, University of Duisburg, preprint SM-DU-211,1993.

[Laur] P.-J. Laurent, Approximation et Optimisation. Hermann, Paris, 1972. (Russian transl. "Mir", Moscow, 1975) MR 57:6947

[LiMa] J.-L. Lions and E. Magenes, Problèmes aux limites non homogènes et applications, vol. I, Dunod, Paris, 1968. MR 40:513

[Ma] W. R. Madych, Polyharmonic cardinal splines, Jour. Approx. Theory, 60 (1990), No. 2, 141-156. MR 91j: 41022

[Mikh] S. Mikhlin, The Problem of the Minimum of a Quadratic Functional, GITTL, MoscowLeningrad, 1952 (English transl. Holden-Day, Inc., San Francisco, London, Amsterdam, 1965) MR 16:41b; MR 30:1427

[Nec] J. Nečas, Les méthodes directes en théorie des équations elliptiques, Academia, Prague, 1967. MR 37:3168

[Schum] L. L. Schumaker, Spline Functions: Basic Theory, J. Wiley and Sons, N.Y., ChichesterBrisbane-Toronto, 1981. MR 82j:41001

[Schw] L. Schwartz, Theorie des distributions, vols. I,II, Paris: Hermann 1950-51 (2nd ed., Ann. Inst. Fourier (Grenoble) 7 (1957), 1-141; 8 (1958), 1-209). MR 12:31d; MR 12:833d; MR 21:6534; MR 22:8322

[Smi] M. H. F. Smith, P. Wessel, Gridding with continuous curvature splines in tension, Geophysics, 55 (1990), No. 3, 293-305.

[Vek] I. N. Vekua, New Methods for Solving Elliptic Equations, OGIZ, Moscow, 1948 (English transl., North-Holland, Amsterdam, and Wiley, New York, 1967). MR 11:598a; MR 35:3243

[Wa] G. Wahba, Surface fitting with scattered noisy data in Euclidean $d$-space and on the sphere, Rocky Mount. J. Math., 14 (Winter 1984), No. 1, pp. 281-299. MR 86c:65017

Institute of Mathematics, Bulgarian Academy of Sciences, Acad. G. Bonchev St. 8, 1113 Sofia, Bulgaria

Department of Mathematics, University of Duisburg, Lotharstr. 65, 4100 Duisburg, GERMANY

E-mail address: kounchev@math.uni-duisburg.de 